\title{
Closure and Consistency In Logic-Associated Argumentation
}

\author{
Phan Minh Dung \\ Phan Minh Thang \\ Computer Science and Information Management Program \\ Asian Institute of Technology \\ GPO Box 4, Klong Luang, Pathumthani 12120, Thailand
}

\author{
DUNG.PHANMINH@GMAIL.COM \\ THANGFM@GMAIL.COM
}

\begin{abstract}
Properties like logical closure and consistency are important properties in any logical reasoning system. Caminada and Amgoud showed that not every logic-based argument system satisfies these relevant properties. But under conditions like closure under contraposition or transposition of the monotonic part of the underlying logic, ASPIC-like systems satisfy these properties. In contrast, the logical closure and consistency properties are not well-understood for other well-known and widely applied systems like logic programming or assumption based argumentation. Though conditions like closure under contraposition or transposition seem intuitive in ASPIC-like systems, they rule out many sensible ASPIC-like systems that satisfy both properties of closure and consistency.

We present a new condition referred to as the self-contradiction axiom that guarantees the consistency property in both ASPIC-like and assumption-based systems and is implied by both properties of closure under contraposition or transposition. We develop a logicassociated abstract argumentation framework, by associating abstract argumentation with abstract logics to represent the conclusions of arguments. We show that logic-associated abstract argumentation frameworks capture ASPIC-like systems (without preferences) and assumption-based argumentation. We present two simple and natural properties of compactness and cohesion in logic-associated abstract argumentation frameworks and show that they capture the logical closure and consistency properties. We demonstrate that in both assumption-based argumentation and ASPIC-like systems, cohesion follows naturally from the self-contradiction axiom. We further give a translation from ASPIC-like systems (without preferences) into equivalent assumption-based systems that keeps the self-contradiction axiom invariant.
\end{abstract}

\section{Introduction}

Properties like logical closure and consistency are important properties in any logical reasoning system. Caminada and Amgoud (2007) showed that not every logic-based argument system satisfies these relevant properties. But under conditions like closure under contraposition or transposition of the monotonic part of the underlying logic, ${ }^{1}$ these properties are fulfilled for ASPIC systems. Prakken (2010) and later Modgil and Prakken (2013) have developed this idea further for ASPIC+, a rich and complex logic-based argumentation system.

The following example illustrates that there are many sensible ASPIC or assumptionbased systems that satisfy both properties of closure and consistency but are neither closed under contraposition nor under transposition.

1. A precise definition of closure under contraposition and transposition is given in section 4 . 
Example 1 Consider an ASPIC-like system $A S=(R S, R D)$ where

1. $R D=R D_{0} \cup R D_{1}$ is a set of defeasible rules and

(a) $R D_{0}$ consists of two defeasible rules

$$
d_{1}: p \Rightarrow \neg f \quad d_{2}: b \Rightarrow f
$$

representing defaults "birds fly, penguins don't" and

(b) $R D_{1}$ consists of a single defeasible rule

$$
t h \Rightarrow b h
$$

representing the default "Thais normally have black hair"

2. $R S=R S_{0} \cup R S_{1}$ is a set of strict rules and

(a) $R S_{0}$ consists of three strict rules

$$
\rightarrow p \quad p \rightarrow b \quad p \rightarrow \neg O j\left(d_{2}\right)
$$

where $\neg O j\left(d_{2}\right)$ means that rule $d_{2}$ is not applicable,

(b) $R S_{1}$ consists of a single strict rule

$$
\rightarrow t h
$$

It is not difficult to see that AS satisfies both properties of closure and consistency.

Even though $A S$ is neither closed under contraposition nor under transposition, there is no reason to rule out systems like AS from consideration as long as they capture our intuition in the concerned applications. In fact, systems like AS often offer a more natural representation of the concerned applications than those closed under contraposition or transposition. To illuminate this point, imagine another ASPIC-like system AS' containing the strict and defeasible rules in $A S$ but is closed under contraposition. ${ }^{2}$

Suppose we are interested in the colour of the hair of the concerned Thai individual. Consider the argument $A: \rightarrow$ th $\Rightarrow$ bh. Let $B_{1}: \rightarrow p \Rightarrow \neg f$ and $B_{2}: \rightarrow p \rightarrow b \Rightarrow f$. Let $C N$ be the consequence operator defined by the strict rules ${ }^{3}$ in $A S^{\prime}$. From $f \in C N(\{f, b h\})$, it follows from the closure under contraposition property of $C N, \neg b h \in C N(\{f, \neg f\})$. Therefore there is an argument $B$ with conclusion $\neg$ bh that contains $B_{1}, B_{2}$ as subarguments. $B$ hence attacks $A$. As $B$ is attacked (by undercut) by argument $C: \rightarrow p \rightarrow \neg O j\left(d_{2}\right)$ at $B_{2}$ and there is no attack against $C, A$ is accepted in the grounded extension. But the set $\{A\}$ itself is not admissible. In other words, to draw a conclusion about the hair colour of Thais, the system needs to resolve a completely unrelated controversy about the flying capabilities of penguins and birds.

2. For example, by adding to $A S$ "absurdity rules" representing the proposition "inconsistency implies every thing", of the form $a, \neg a \rightarrow l$ where a is an atom appearing in $R S \cup R D$ and $l$ is a literal over this set of atoms. It is not difficult to see that $A S^{\prime}$ is closed under contraposition (see appendix of section 1 ). 3. A precise definition is given in definition 1. 


\section{Closure and Consistency In Logic-Associated Argumentation}

In general, the condition of closure under contraposition creates an attack against any defeasible argument from any inconsistency in ASPIC systems. In other words, if the systems happen to contain knowledge about several causally independent domains like about the flying penguins and the hair colour of Thais, closure under contraposition will interlink them and making it necessary to resolve all possible inconsistencies in all parts of the systems before being able to answer any query, independent of whether these inconsistencies are causally related to the query or not. ${ }^{4}$

In contrast, in argument systems like $A S$, there is no attack against $A$ and $\{A\}$ is admissible. Controversy about the flying capabilities of penguins do not have any effect on the acceptance of $A$.

Though closure under transposition avoids the problem of conflict propagation, it is not adopted in many well-known and practical systems as an indiscriminate application of it could lead to counter-intuitive result in many cases. For example, consider a simplified version of example "birds fly penguins don't" in aspic+ whose set of strict rules is the closure under transposition of the following strict rules:

$$
a p p, b \rightarrow f \quad p \rightarrow \neg f \quad p \rightarrow b \quad p \rightarrow \neg a p p
$$

where app is an ordinary premise with $\overline{\text { app }}=\neg$ app.

Therefore the rule "app $\rightarrow \neg p$ " is included in the set of strict rules.

Suppose the knowledge base only consists of the ordinary premise app stating that the default "birds fly" is applicable.

Without any further information, the only two arguments are: $A \equiv$ app and $B \equiv$ $A \rightarrow \neg p$.

Accepting ordinary premise app simply says that there is no information at all why the default "birds fly" should not be applied. It does not say anything about penguin. But closure under transposition implies that there is definitely no penguin around (a rather slippery way to make a conclusion).

In the context of logic programming, the rule "app $\rightarrow \neg p$ " is also rather unnatural as app is viewed as an assumption and $\neg$ is the explicit negation operator whose intuitive reading is that an explicit negation of an assertion should be based on some "hard evidence", not on another assumption (Gelfond E Lifschitz, 1990).

In contrast to ASPIC systems, not much research has been done to study the logical closure and consistency of the semantics of other well-known and widely applied systems like logic programming or assumption based argumentation (Gelfond \& Lifschitz, 1988; Lifschitz, 1999; Bondarenko, Dung, Kowalski, \& Toni, 1997). Properties like closure under transposition or contraposition are not embraced in logic programming or assumption based argumentation. Prakken (2012) has observed that assumption-based argumentation yields unintuitive argument with unwanted conclusion if the inference rules satisfy the transposition property. It is hence natural to ask

whether there are conditions that are implied by both conditions of closure under contraposition and transposition but still guaranteeing the important properties of logical closure and consistency, and

4. Pollock (1995) and $\mathrm{Wu}(2012)$ have also pointed out that classical propositional proof systems propagate conflicts throughout the knowledge bases to unrelated parts. 
whether the logical closure and consistency properties could be stated and studied in a general framework generalizing both assumption-based and ASPIC-like systems and possibly also other logic-based argument systems?

We will present in this paper a new condition referred to as the self-contradiction axiom ${ }^{5}$ that guarantees the consistency of complete extension semantics and is implied by both conditions of closure under contraposition or transposition while at the same time, also includes systems that could avoid the problem of conflict-propagation. In example 1 both $A S, A S^{\prime}$ satisfy the self-contradiction axiom.

It turns out that the consistency and closure properties in logic-based argument systems could be studied within a general logic-associated abstract argumentation framework, obtained by associating abstract argumentation with abstract logics to represent the conclusions of arguments. We demonstrate that logic-associated abstract argumentation frameworks capture ASPIC-like systems (without preferences) and assumption-based argumentation. We present two simple and natural properties of compactness and cohesion in logic-associated abstract argumentation frameworks and show that they capture the logical closure and consistency of the complete extension semantics. We demonstrate that in both assumption-based argumentation and ASPIC-like systems cohesion follows naturally from the self-contradiction axiom.

We further give a translation from ASPIC-like systems (without preferences) into equivalent assumption-based systems that keeps the self-contradiction property invariant.

The paper is structured as follows. In section 2 we recall abstract argumentation and Tarski abstract logics. We then introduce in section 3 a framework in which abstract argumentation is associated to abstract logics where sentences in abstract logics represent argument conclusions. We present in this section two simple and natural conditions of compactness and cohesion and show that they ensure the satisfaction of the properties of logical closure and consistency of complete extensions. In sections 4,5 we show how compactness and cohesions could be captured naturally in ASPIC-like systems or assumption-based argumentation. We introduce in section 4 the fundamental axiom of self-contradiction and show the connections between it and the properties of closure under contraposition and transposition. We provide in section 6 a transformation from ASPIC-like systems into equivalent assumption-based framework. In section 7 , we discuss the more recent systems based on Tarski's abstract logics. We then conclude. ${ }^{6}$

\section{Preliminaries}

An abstract argumentation framework (Dung, 1995) is defined simply by a pair ( $A R$, att) of a set of arguments $A R$ and att $\subseteq A R \times A R$ where $(A, B) \in$ att represents an attack from argument $A$ against argument $B$. A set of argument $S$ attacks an argument $A$ if some argument in $S$ attacks $A$. S attacks another set $S^{\prime}$ if $\mathrm{S}$ attacks an argument in $S^{\prime} . S$ is conflict-free if it does not attack itself. $S$ is conflicting if it attacks itself. An argument $\mathrm{A}$ is acceptable wrt set of arguments $\mathrm{S}$ if $\mathrm{S}$ attacks each attack against $\mathrm{A} . S$ is admissible if $S$ is conflict-free and it counter-attacks each attack against it. The semantics

5. The intuitive reading of "self-contradiction" is that if "X causes contradiction" then "X contradicts itself".

6. A very preliminary extended abstract of this paper is published by Dung and Thang (2011). 
of abstract argumentation is determined by the acceptability of arguments and various associated notions of extensions. A complete extension is an admissible set containing every argument acceptable wrt it. Complete extensions could also be viewed as conflict-free fixed points of the characteristic function $F: 2^{A R} \rightarrow 2^{A R}$ defined by $F(S)=$ set of acceptable arguments wrt S. Preferred extensions are maximal conflict-free fixed points of $\mathrm{F}$ while the least fixed point of $\mathrm{F}$ is called the grounded extension. While there could be many preferred extensions, there exists an unique grounded extension. A stable extension is a conflict-free set of arguments that attacks every argument not belonging to it.

Amgoud and Besnard (2009) have proposed the use of Tarski's abstract logic in argumentation that is characterized simply by a consequence operator.

Given a language $\mathcal{L}$ of well-formed formulas, a Tarski abstract logic (Amgoud \& Besnard, 2009 ) is defined by a consequence operator $\mathrm{CN}: 2^{\mathcal{L}} \rightarrow 2^{\mathcal{L}}$ such that following axioms are satisfied:

1. (Expansion)

2. (Idempotence)

3. (Finiteness)

4. (Absurdity)

5. (Coherence)
$X \subseteq C N(X)$

$C N(C N(X))=C N(X)$

$C N(X)=\bigcup\{C N(Y) \mid Y \subseteq X$ and $Y$ is finite $\}$

$C N(\{x\})=\mathcal{L}$ for some $x \in \mathcal{L}$

$C N(\emptyset) \neq \mathcal{L}$

We introduce below the consequence operator over a set of strict or inference rules.

A strict rule $^{7}$ is of the form

$$
\alpha_{1}, \ldots, \alpha_{n} \rightarrow \alpha
$$

where $\alpha_{1}, \ldots, \alpha_{n}, \alpha$ are from $\mathcal{L}$.

Definition 1 Let $R S$ be a set of strict rules. Define the consequence operator $C N_{R S}$ : $2^{\mathcal{L}} \rightarrow 2^{\mathcal{L}}$ as follows: For a set $X \subseteq \mathcal{L}, C N_{R S}(X)$ is the smallest set such that

1. $X \subseteq C N_{R S}(X)$, and

2. for each rule $\sigma_{1}, \ldots \sigma_{n} \rightarrow \sigma$ in $R S$, if $\left\{\sigma_{1}, \ldots \sigma_{n}\right\} \subseteq C N_{R S}(X)$ then $\sigma \in C N_{R S}(X)$.

\section{Associating Abstract Argumentation With Abstract Logics}

Intuitively, an argument is a "proof" of some conclusion. In many cases, such proofs are constructed following some proof theory of some formal logics. Such logics could be nonmonotonic. The notions of closure and consistency are then defined according to the monotonic parts of the underlying logics.

Many logics underlying argumentation systems like assumption-based argumentation or ASPIC-systems do not always impose the absurdity axiom. This motivates our slight generalization of Tarski abstract logics in the following definition.

7. often also referred to as inference rules in assumption-based argumentation. 
Definition 2 Given a language $\mathcal{L}$, an abstract logic is defined as a pair $(C N, C O N T R A)$ where $C N: 2^{\mathcal{L}} \rightarrow 2^{\mathcal{L}}$ represents a consequence operator and $C O N T R A \subseteq 2^{\mathcal{L}}$ is a collection of contradictory sets such that following axioms hold:

1. CN satisfies the expansion, idempotence and finiteness axioms.

2. (Weak Absurdity) If $S \in C O N T R A$ then each superset of $S$ also belongs to CONTRA. ${ }^{8}$

3. (Weak Coherence) $C N(\emptyset) \notin C O N T R A$.

Given an abstract logic $(C N, C O N T R A), \mathrm{X}$ is closed iff $X=C N(X)$. A set $X \in$ $C O N T R A$ is said to be contradictory.

A set $X \subseteq \mathcal{L}$ is said to be inconsistent iff its closure $C N(X)$ is contradictory. ${ }^{9} \mathrm{X}$ is said to be consistent iff it is not inconsistent. $\mathrm{X}$ is minimal inconsistent iff $\mathrm{X}$ is inconsistent and each proper subset of $\mathrm{X}$ is consistent. ${ }^{10}$

Definition 3 We say that an abstract logic satisfies the

strong absurdity axiom if $C O N T R A \neq \emptyset$ and for each $X \in C O N T R A, C N(X)=\mathcal{L}$.

It follows immediately that an abstract logic is a Tarski abstract logic if it satisfies the strong absurdity axiom and there is $x \in \mathcal{L}$ such that $\{x\}$ is inconsistent. ${ }^{11}$

Example 2 Let $R S_{0}=\{\rightarrow w r ; \rightarrow g o ; b \rightarrow \neg h w ; m \rightarrow h w\}$ be a set of strict rules, ${ }^{12}$ and $\mathcal{L}$ be a language consisting of literals whose atoms occur in the rules in $R S_{0}$. Define $A L_{0}=\left(C N_{0}, C O N T R A\right)$ as follows:

- $X \in C O N T R A$ iff $X$ contains a pair of literals $\{l, \neg l\}$.

- $C N_{0}$ is the consequence operator defined by $R S_{0}$.

For illustration, $C N_{0}(\emptyset)=\{w r, g o\}, C N_{0}(\{m\})=\{w r, g o, m, h w\}$ and $C N_{0}(\{m, b\})=$ $\{w r, g o, m, h w, b, \neg h w\}$. Hence the set $\{m, b\}$ is inconsistent but not contradictory.

It is not difficult to see that $A L_{0}$ is an abstract logic. From $C N_{0}(\{h w, \neg h w\})=$ $\{h w, \neg h w\}$, it follows that $A L_{0}$ does not satisfy the strong absurdity axiom.

8. Note that $C O N T R A$ could be empty like in the case of definite logic programs where $\mathcal{L}$ consists only of positive literals. Nonetheless, if $C O N T R A \neq \emptyset$ then $\mathcal{L} \in C O N T R A$.

9. In other words, a set of sentences $\mathrm{X}$ is inconsistent if a contradiction could be derived from it though contradiction may not be present directly in $\mathrm{X}$ (i.e. an inconsistent set may not be contradictory).

10. $\mathrm{Y}$ is a proper subset of $\mathrm{X}$ if $\mathrm{Y}$ is a subset of $\mathrm{X}$ and $Y \neq X$.

11. To be a Tarskian one, an abstract logic needs to satisfy absurdity and coherence. Because $\{x\}$ is inconsistent, $C N(\{x\})$ is contradictory. Since strong absurdity is satisfied, $C N(C N(\{x\}))=L$. Because of idempotence, $C N(C N(\{x\}))=C N(\{x\})$. Hence $C N(\{x\})=L$. So coherence holds.

From the weak coherence axiom of abstract logic, $C N(\emptyset) \notin C O N T R A$. Due to the strong absurdity,

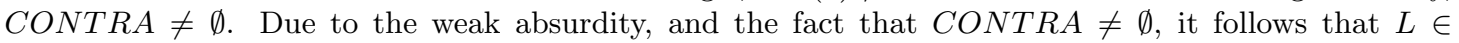
$C O N T R A$. Hence $C N(\emptyset) \neq L$.

12. The rules are taken from an example by Caminada and Amgoud (2007) where wr = "John wears something that looks like a a wedding ring", $\mathrm{m}=$ "John is married", $\mathrm{hw}=$ "John has a wife", go = "John often goes out until late", b = "John is a bachelor". 
Inspired by Amgoud and Besnard's idea (2009), we use abstract logics to represent the conclusions of arguments but in difference to them, we do not specify in detail the structure of individual arguments.

Definition $4 A$ logic-associated argumentation framework over a language $\mathcal{L}$ is a quadruple $(A F, \sqsubseteq, A L, C n l)$ where

1. $A F=(A R, a t t)$ is an abstract argumentation framework, and

2. $A L=(C N, C O N T R A)$ is an abstract logic over $\mathcal{L}$, and

3. $C n l: A R \rightarrow \mathcal{L}$ assigns to each argument $A$, its conclusion $C n l(A)$ in $\mathcal{L}$, and

4. $\sqsubseteq$ is a partial order ${ }^{13}$ over $A R$ where $A \sqsubseteq B$ means that $A$ is a subargument of $B$ such that for all arguments $C \in A R$, if $C$ attacks $A$ then $C$ attacks $B$.

Remark 1 - For a set $S$ of arguments, $\mathbf{C n l}(\mathbf{S})$ denotes the set of the conclusions of the arguments in $S$.

- The set of all subarguments of $A$ is denoted by $\mathbf{S u b}(\mathbf{A})$. For a set of arguments $S$, $\mathbf{S u b}(\mathbf{S})$ contains all subarguments of arguments in $S$.

We next give an example of a logic-associated argumentation framework.

Example 3 Let $L A F_{0}=\left(A F_{0}, \sqsubseteq_{0}, A L_{0}, C n l\right)$ where $A L_{0}$ is defined in example 2 and $A F_{0}=\left(A R_{0}, a t t_{0}\right)$ such that

1. The arguments in $A R_{0}$ are constructed with rules from the set of strict rules $R S_{0}$ in example 2, and a set of defeasible rules $R D=\{w r \Rightarrow m ;$ go $\Rightarrow b\}$. There are 6 arguments $^{14}$ :

$A_{1}: \rightarrow w r, A_{3}: \rightarrow w r \Rightarrow m, \quad A_{5}: \rightarrow w r \Rightarrow m \rightarrow h w$.

$A_{2}: \rightarrow g o, \quad A_{4}: \rightarrow g o \Rightarrow b, \quad A_{6}: \rightarrow g o \Rightarrow b \rightarrow \neg h w$.

Attack relation: $A_{5}$ attacks $A_{6}$ and vice versa. There are no other attacks. Let att $_{0}=\left\{\left(A_{5}, A_{6}\right),\left(A_{6}, A_{5}\right)\right\}$.

2. The subargument relation $\sqsubseteq_{0}$ is the reflexive and transitive closure of $A_{1} \sqsubseteq A_{3} \sqsubseteq A_{5}$ and $A_{2} \sqsubseteq A_{4} \sqsubseteq A_{6}$.

Definition 5 Let $L A F=(A F, \sqsubseteq, A L, C n l), A L=(C N, C O N T R A)$, be a logic-associated argumentation framework.

1. LAF is said to satisfy the closure-property if for each complete extension $E$ of $A F$, $\operatorname{Cnl}(E)$ is closed wrt AL.

13. A partial order is a reflexive, asymmetric and transitive relation.

14. For a precise definition see definition 12 . 
2. $L A F$ is said to satisfy the consistency-property if for each complete extension $E$ of $A F, C n l(E)$ is consistent wrt $A L$.

Example 4 (Continuation of example 3)

The grounded extension of $A F_{0}$ is $G E=\left\{A_{1}, A_{2}, A_{3}, A_{4}\right\}$. There are two preferred extensions $E_{1}=\left\{A_{1}, A_{2}, A_{3}, A_{4}, A_{5}\right\}$ and $E_{2}=\left\{A_{1}, A_{2}, A_{3}, A_{4}, A_{6}\right\}$.

$C n l(G E)=\{w r, g o, m, b\}$ and $C N_{0}(C n l(G E))=C n l(G E) \cup\{h w, \neg h w\}$. Hence the set of conclusions of arguments in the grounded extension are neither closed nor consistent wrt the abstract logic $A L_{0}$. Hence $L A F_{0}$ satisfies neither the closure-property nor the consistency-property.

It is also easy to see that the sets of conclusions of the arguments of the two preferred extensions are neither closed nor consistent either.

Example 5 (Continuation of example 4)

Let $R S_{1}=R S_{0} \cup\{\neg h w \rightarrow \neg m, \quad h w \rightarrow \neg b\}$. The consequence operator wrt $R S_{1}$ is denoted by $C N_{1}$. Let $A L_{1}=\left(C N_{1}, C O N T R A\right)$ and $A F_{1}=\left(A R_{1}\right.$, att 1$)$ where

- $A R_{1}=A R_{0} \cup\left\{A_{7}, A_{8}\right\}$ with $A_{7}: A_{5} \rightarrow \neg b$ and $A_{8}: A_{6} \rightarrow \neg m,{ }^{15}$

- $\operatorname{att}_{1}=\left\{\left(A_{7}, A_{4}\right),\left(A_{7}, A_{6}\right),\left(A_{7}, A_{8}\right),\left(A_{8}, A_{3}\right),\left(A_{8}, A_{5}\right),\left(A_{8}, A_{7}\right)\right\}$,

- $\sqsubseteq_{1}$ is the reflexive and transitive closure of $\sqsubseteq_{0} \cup\left\{A_{5} \sqsubseteq A_{7}, A_{6} \sqsubseteq A_{8}\right\}$.

The grounded extension of $A F_{1}$ is $G E^{\prime}=\left\{A_{1}, A_{2}\right\}$. Two preferred extensions of $A F_{1}$ are $E_{1}^{\prime}=\left\{A_{1}, A_{2}, A_{3}, A_{5}, A_{7}\right\}$ and $E_{2}^{\prime}=\left\{A_{1}, A_{2}, A_{4}, A_{6}, A_{8}\right\}$.

It is not difficult to see that the sets $C n l\left(G E^{\prime}\right)=\{w r, g o\}, C n l\left(E_{1}^{\prime}\right)=\{w r, g o, m, h w, \neg b\}$, $\operatorname{Cnl}\left(E_{2}^{\prime}\right)=\{w r, g o, b, \neg h w, \neg m\}$ are closed and consistent.

Let $L A F_{1}=\left(A F_{1}, \sqsubseteq_{1}, A L_{1}, C n l\right)$. Both closure and consistency properties are satisfied in $L A F_{1}$.

From now on until the end of this section, we assume an arbitrary but fixed logicassociated framework $L A F=(A F, \sqsubseteq, A L, C n l)$.

It turns out that both the closure and consistency properties are based on an intuitive idea of a base of an argument.

Definition 6 Let $A$ be an argument and $B A$ be a finite set of subarguments of $A$. BA is said to be a base of $A$ if following conditions are satisfied:

1. $\operatorname{Cnl}(A) \in C N(C n l(B A))$

2. For each argument $C, C$ attacks $A$ iff $C$ attacks $B A$.

It is easy to see that for each argument $\mathrm{A},\{A\}$ is a base of $\mathrm{A}$.

In example 3, though $C n l\left(A_{5}\right) \in C N_{0}\left(C n l\left(A_{3}\right)\right),\left\{A_{3}\right\}$ is not a base of $A_{5}$ since $A_{6}$ attacks $A_{5}$ but $A_{6}$ does not attack $A_{3}$.

Note that the empty set is a base of both arguments $A_{1}$ and $A_{2}$.

In contrast, in example $5,\left\{A_{3}\right\}$ is a base of $A_{5}$ and $A_{7}$ and $\left\{A_{4}\right\}$ is a base of $A_{6}$ and $A_{8}$.

15. i.e. $A_{7} \equiv \rightarrow w r \Rightarrow m \rightarrow h w \rightarrow \neg b$, and $A_{8} \equiv \rightarrow g o \Rightarrow b \rightarrow \neg h w \rightarrow \neg m$. 
Definition 7 1. An argument $A$ is said to be generated by a set of arguments $S$ if there is a base $B A$ of $A$ such that $B A \subseteq S u b(S)$.

2. The set of all arguments generated by $S$ is denoted by $\mathbf{G N}(\mathbf{S})$.

It follows immediately

Lemma 1 Let $S$ be a set of arguments. The following assertions hold:

1. For each argument $A, A$ is generated by $\{A\}$.

2. $\operatorname{Sub}(S) \subseteq G N(S)$.

3. $C n l(G N(S)) \subseteq C N(C n l(S u b(S)))$.

4. $C N(C n l(G N(S)))=C N(C n l(S u b(S)))$.

5. For each argument $C, C$ attacks $G N(S)$ iff $C$ attacks $S$.

Proof See appendix of section 3.

Theorem 1 Let $E$ be a complete extension. Then $G N(E)=E$

Proof See appendix of section 3.

Theorem 1 motivates the following definitions 8, 9 .

Definition 8 We say that a logic-associated argumentation framework $L A F$ is compact if for each set of arguments $S, C n l(G N(S))$ is closed.

The argumentation framework in example 3 is not compact since for $S=\left\{A_{3}\right\}, G N(S)=$ $\left\{A_{1}, A_{2}, A_{3}\right\}$ and $C n l(G N(S))=\{w r, g o, m\}$ is not closed since $C N(\{w r, g o, m\})=\{w r, g o, m, h w\}$.

In contrast, the argumentation framework in example 5 is compact. For example, $G N(S)=\left\{A_{1}, A_{2}, A_{3}, A_{5}, A_{7}\right\}$ (wrt $\left.L A F_{1}\right)$ and $\operatorname{Cnl}(G N(S))=\{w r, g o, m, h w, \neg b\}$ is both closed and consistent.

Theorem 2 Each compact logic-associated argumentation framework satisfies the closureproperty.

Proof. Let E be a complete extension. From the compactness, it follows that $\operatorname{Cnl}(G N(E))$ is closed. From theorem 1, $\mathrm{Cnl}(E)$ is closed.

From lemma 1, it follows that LAF is compact iff $C n l(G N(S))=C N(C n l(S u b(S)))$ iff $C N(C n l(S u b(S))) \subseteq C n l(G N(S))$. We have proved

Lemma 2 LAF is compact iff for each set $S$ of arguments, $C N(C n l(S u b(S))) \subseteq C n l(G N(S))$

Notation 1 Abusing the notations for simplicity, we often refer to a set of arguments $\mathbf{S}$ as inconsistent (resp. consistent) if $C n l(S u b(S))$ is inconsistent (resp. consistent). 
Intuitively, inconsistency among a set of arguments indicates a possible conflict among its generated arguments. This insight motivates the following definitions.

Definition 9 A logic-associated argumentation framework $L A F$ is said to be cohesive if for each inconsistent set of argument $S, G N(S)$ is conflicting.

For illustration, consider the logic-associated argumentation framework in example 3. Let $S=\left\{A_{3}, A_{4}\right\}$. It is clear that $\mathrm{S}$ is inconsistent. It is not difficult to see that each base of each argument $A \in\left\{A_{5}, A_{6}\right\}$ contains argument $A$ itself. Therefore $G N(S)=$ $\left\{A_{1}, A_{2}, A_{3}, A_{4}\right\} . G N(S)$ is thus conflict-free. The framework $L A F_{0}$ is hence not cohesive.

Note that the argumentation framework $L A F_{1}$ in example 5 is both compact and cohesive.

Theorem 3 Let LAF be a cohesive logic-associated argumentation framework. Then LAF satisfies the consistency-property.

Proof Let E be a complete extension. Suppose $C n l(E)$ is inconsistent. From the cohesion of LAF, it follows that $G N(E)$ is conflicting. From theorem 1, E is conflicting. Contradiction. Hence $C n l(E)$ is consistent.

It follows immediately from theorems 2 and 3:

Corollary 1 Let LAF be a compact and cohesive logic-associated argumentation framework. Then LAF satisfies both the closure- and consistency-properties.

In the next sections, we show that ASPIC-like systems (without preferences) and assumptionbased argumentation are instances of logic-associated argumentation frameworks. We will also introduce the axiom of self-contradiction to guarantee the cohesion (and consistency) of these systems.

\section{Argumentation with Strict and Defeasible Rules}

We assume a language $\mathcal{L}$ of literals where a literal is an atom $a$ or the explicit negation $\neg a$ of atom a. A set of literals is said to be contradictory if it contains a pair $a, \neg a$.

It is important to note that we identify $\neg \neg a$ with a. For $X \subseteq \mathcal{L}$, denote $\neg X=\{\neg l \mid l \in$ $X\}$.

A defeasible rule is of the form

$$
\alpha_{1}, \ldots, \alpha_{n} \Rightarrow \alpha
$$

where $\alpha_{1}, \ldots, \alpha_{n}, \alpha$ are from $\mathcal{L}$.

Definition $10 A$ rule-based argumentation system is a pair $A S=(R S, R D)$ of a set $R S$ of strict rules and a set $R D$ of defeasible rules such that $C N_{R S}(\emptyset)$ is not contradictory.

The following definition 11 identifies the abstract logic underlying a rule-based argumentation system $A S=(R S, R D)$. 
Definition 11 Let $A S=(R S, R D)$ be a rule-based argumentation system. Define

$$
A L_{A S}=\left(C N_{A S}, C O N T R A_{A S}\right)
$$

where $C O N T R A_{A S}$ is the collection of all contradictory sets and $C N_{A S}=C N_{R S}$.

It follows immediately

Lemma $3 A L_{A S}$ is an abstract logic.

We recall below the arguments and attack relations of rule-based argumentation systems introduced by Caminada and Amgoud (2007), Prakken (2010), Pollock (1987), and Modgil and Prakken (2013).

Definition 12 1. Rules of the form $\rightarrow / \Rightarrow \alpha$, are arguments with conclusion $\alpha$.

2. Let $r$ be a strict/defeasible rule of the form $\alpha_{1}, \ldots, \alpha_{n} \rightarrow / \Rightarrow \alpha, n \geq 0$. Further suppose that $A_{1}, \ldots, A_{n}, n \geq 0$, are arguments with conclusions $\alpha_{1}, \ldots, \alpha_{n}$ respectively. Then $A_{1}, \ldots, A_{n} \rightarrow / \Rightarrow \alpha$ is an argument with conclusion $\alpha$ and last rule $r$.

3. Every argument is constructed by applying finitely many times the above two steps.

We next introduce key notations.

Notation 2 1. A strict argument is an argument containing no defeasible rule. Nonstrict arguments are called defeasible arguments.

2. A basic defeasible argument is an argument whose last rule is a defeasible one, i.e. of the form $A_{1}, \ldots, A_{n} \Rightarrow \alpha$.

For a basic defeasible argument $B$, the last rule of $B$ is denoted by $\mathbf{L r}(\mathbf{B})$.

3. $B$ is a subargument of an argument $A$ of the form $A_{1}, \ldots, A_{n} \rightarrow / \Rightarrow \alpha$, denoted by $B \sqsubseteq A$, if $B=A$ or $B$ is a subargument of some $A_{i}$.

Remark 2 The conclusion of an argument $A$ is denoted by $\operatorname{Cnl}(A)$.

Remark 3 Arguments of the form $A_{1}, \ldots, A_{n} \rightarrow / \Rightarrow \alpha$ are also often viewed as proof trees with the root labelled by $\alpha$ and the children of the root are the roots of subtrees $A_{1}, \ldots, A_{n}$. Note that if $n=0$, the proof tree consists of just the root.

Illustrations for argumentation systems based on strict and defeasible rules are given in examples 3,5 .

The following notion of attack is adopted from articles of Caminada and Amgoud (2007), Prakken (2010), Pollock (1987), and Modgil and Prakken (2013).

Definition 13 An argument $A$ attacks an argument $B$ (on $B^{\prime}$ ) if $B^{\prime}$ is basic defeasible subargument of $B$ and one of the following conditions is satisfied: 
1. (Undercutting) $C n l(A)=\neg O j\left(\operatorname{Lr}\left(B^{\prime}\right)\right)$ where for a defeasible rule $r, O j(r)$ is an atom denoting that rule $r$ is applicable.

2. (Rebutting) $\operatorname{Cnl}(A)=\neg \operatorname{Cnl}\left(B^{\prime}\right)$.

Remark 4 For simplicity, we identify a rule-based argumentation system AS with the logicassociated argumentation framework $\left(A F_{A S}, \sqsubseteq, A L_{A S}, C n l\right)$ where $A F_{A S}$ is the argumentation framework obtained from AS according to definitions 12,13.

Theorem 4 Rule-based argumentation systems are compact.

Proof See appendix of section 4 .

The following lemma reveals an simple but important relation between an arguments and its basic defeasible subarguments.

Lemma 4 Let $A$ be an argument and $B D$ be the set of basic defeasible subarguments of $A$. Then $\operatorname{Cnl}(A) \in C N_{A S}(C n l(B D))$.

Proof See appendix of section 4 .

We introduce now a fundamental condition underlying the cohesion of rule-based argumentation.

Definition 14 The abstract logic $A L_{A S}$ is said to satisfy the self-contradiction axiom if for each minimal inconsistent set $X \subseteq \mathcal{L}: \neg X \subseteq C N_{A S}(X) .{ }^{16}$

The example below illustrates the intuition of the self-contradiction axiom using again the famous "birds fly penguins don't"-example.

Example 6 Let $A S=(R S, R D)$ where $R D$ consists of two defeasible rules:

$$
d_{1}: \quad p \Rightarrow \neg f \quad d_{2}: \quad b \Rightarrow f
$$

and $R S$ consists of three strict rules

$$
r_{0}: \rightarrow p \quad r_{1}: p \rightarrow b \quad r_{2}: p \rightarrow \neg O j\left(d_{2}\right)
$$

where $O j\left(d_{2}\right)$ is an atom stating that rule $d_{2}$ is applicable.

It is obvious that the set of strict rules $R S$ is not closed under transposition. It is also straightforward to see that $C N_{A S}(\{f, \neg f\})=\{f, \neg f\} \neq \mathcal{L}$. Hence the underlying abstract logic $A L_{A S}$ satisfies neither the strong absurdity axiom nor the closure under transposition property. ${ }^{17}$

16. The intuitive reading of "self-contradiction" is that if "X causes contradiction" then "X contradicts itself".

17. See section 4.1 for precise definitions of closure under transposition or contraposition and their relationships with strong absurdity and self-contradiction 
It is not difficult to see that $A L_{A S}$ satisfies the self-contradiction axiom. ${ }^{18}$

It is not difficult to see that AS satisfies both properties of closure and consistency.

The following theorem shows that self-contradiction is sufficient for cohesion.

Theorem 5 Suppose $A L_{A S}$ satisfies the self-contradiction axiom. Then $A S$ is cohesive.

Proof See appendix of section 4.

It follows immediately from corollary 1 that

Corollary 2 Suppose $A L_{A S}$ satisfies the self-contradiction axiom. Then AS satisfies both closure- and consistency-properties.

We next relate theorem 5 and corollary 2 to the the results by Caminada and Amgoud (2007), Prakken (2010), and Modgil and Prakken (2013).

\subsection{Sufficient Conditions for Self-Contradiction in Abstract Logics $A L_{A S}$}

For simplicity, if there are no possibilities for misunderstanding, we often write respectively $C N, C O N T R A$ and $A L$ for $C N_{A S}, C O N T R A_{A S}$ and $A L_{A S}$ in this section.

We first recall the definitions of closure under contraposition and transposition from articles of Caminada and Amgoud (2007), Prakken (2010), and Modgil and Prakken (2013).

\section{Definition 15 1. AL is said to be}

closed under contraposition if for each set $X \subseteq \mathcal{L}$, for each $\alpha \in X$ if $\sigma \in C N(X)$ then $\neg \alpha \in C N(X \backslash\{\alpha\} \cup\{\neg \sigma\})$.

2. A set of strict rules $R S$ is said to be

closed under transposition if for each rule $\alpha_{1}, \ldots, \alpha_{n} \rightarrow \sigma$ in $R S$, all the rules of the form $\alpha_{1}, \ldots, \alpha_{i-1}, \neg \sigma, \alpha_{i+1}, \alpha_{n} \rightarrow \neg \alpha_{i}$ also belong to $R S$.

The relations between the closure under contraposition and the axioms of self-contradiction and strong absurdity are illuminated in the following lemma.

Lemma 5 1. If $A L$ is closed under contraposition, then $A L$ satisfies the strong absurdity axiom.

2. If AL satisfies the strong absurdity axiom then AL satisfies the self-contradiction axiom.

18. Let $\mathrm{S}$ be a minimal inconsistent set. We show $\neg S \subseteq C N_{A S}(S)$. If $\mathrm{S}$ is contradictory then the minimality of $\mathrm{S}$ implies that $S=\{a, \neg a\}$ for some $a \in \mathcal{L}$. The self-contradiction axioms holds obviously. Suppose $\mathrm{S}$ is not contradictory. Hence there is a pair $\{a, \neg a\} \subseteq C N_{A S}(S)$ for some atom a. It is not difficult to see that $C N_{A S}(S)=C N_{A S}(\emptyset) \cup S$. Therefore $C N_{A S}(S) \backslash S \subseteq C N_{A S}(\emptyset)=\left\{p, b, \neg O j\left(d_{2}\right)\right\}$. It follows $\{a, \neg a\} \cap C N_{A S}(\emptyset) \neq \emptyset$ and $\{a, \neg a\} \cap S \neq \emptyset$. From the minimality of S, S consists of exactly one element. Therefore $\forall \sigma \in S: \neg \sigma \in C N_{A S}(S)$. 
Proof See appendix of section 4 .

The relations between the closure under transposition and the self-contradiction axiom is illuminated in the following lemma.

Lemma 6 Let $A S=(R S, R D)$ such that the set of strict rules $R S$ is closed under transposition. Then $A L_{A S}$ satisfies the self-contradiction axiom.

Proof See appendix of section 4 .

The following example shows that the reverses of assertions in lemmas 5, 6 do not hold in general.

Example 7 Let $\mathcal{L}=\{a, \neg a, b, \neg b\}$. Let CONTRA be the set of all contradictory sets over $\mathcal{L}$.

1. For each $X \subseteq \mathcal{L}$, define $C N(X)=X$. It is obvious that the abstract logic $A L=$ $(C N, C O N T R A)$ satisfies the self-contradiction axiom but not the strong absurdity axiom.

2. Consider a set of strict rules $R S$ consisting of a "normal" rule $a \rightarrow b$ together with "absurdity rules" of the form $x, \neg x \rightarrow y$ where $x \in\{a, b\}, y \in \mathcal{L}$. Let $C N$ be the consequence operator wrt $R S$.

It is obvious that $A L=(C N, C O N T R A)$ satisfies the strong absurdity axiom (and hence also the self-contradiction axiom). From $b \in C N(\{a\})$, but $\neg a \notin C N(\{\neg b\})=$ $\{\neg b\}$, it follows that $A L$ is not closed under contraposition.

It is clear that the set of strict rules is not closed under transposition.

3. Consider a set of strict rules $R S$ consisting of just two strict rules $a \rightarrow b$ and $\neg b \rightarrow$ $\neg a$. It is clear that the rule set is closed under transposition but the corresponding consequence operator does not satisfy the strong absurdity axiom.

The following picture illustrates the relationships between key properties of rule-based argumentation.

\section{Contra Position $\vDash$ Strong Absurdity

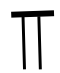

Transposition $\vDash$ Self-Contradiction $\vDash$ Cohesion $\vDash$ Consistency

Figure 1: 


\section{Assumption-Based Argumentation}

Given a logical language $\mathcal{L}$, an assumption-based argumentation (ABA) framework (Bondarenko et al., 1997) is a triple $\mathcal{F}=(\mathcal{R}, \mathcal{A},-)$ where $\mathcal{R}$ is a set of inference rules of the form $\sigma_{1}, \ldots \sigma_{n} \rightarrow \sigma$ (for $n \geq 0$ ), and $\mathcal{A} \subseteq \mathcal{L}$ is a set of assumptions, and - is a (total) one-one mapping from $\mathcal{A}$ into $\mathcal{L}$, where $\bar{x}$ is referred to as the contrary of $x$ such that following properties are satisfied:

- assumptions in $\mathcal{A}$ do not appear in the heads of rules in $\mathcal{R}$, and

- contraries of assumptions are not assumptions, and

- if $\mathcal{L}$ contains an explicit negation operator $\neg$ then $C N_{\mathcal{R}}(\emptyset)$ is not contradictory wrt $\neg$, i.e. for all $\sigma \in \mathcal{L},\{\sigma, \neg \sigma\} \nsubseteq C N_{\mathcal{R}}(\emptyset)$.

The following edition of the "birds fly penguins don't" example provides an illustration.

Example $8 \mathcal{F}=(\mathcal{R}, \mathcal{A},-)$ where $\mathcal{R}$ consists of rules

$$
n o t \_a b_{1}, p \rightarrow \neg f \quad n o t_{-} a b_{2}, b \rightarrow f \quad \rightarrow p \quad p \rightarrow b \quad p \rightarrow a b_{2}
$$

and $\mathcal{A}=\left\{n o t \_a b_{1}, n o t \_a b_{2}\right\} \quad$ and $\overline{n o t \_a b_{1}}=a b_{1}, \overline{n_{\text {not_ab }}}=a b_{2}$

We identify the structure of abstract logics underlying ABA frameworks below.

\subsection{Assumption-based Abstract Logics}

A set $X \subseteq \mathcal{L}$ is said to be contradictory iff

- $\mathrm{X}$ is contradictory wrt - , i.e. there exists an assumption $\alpha \in \mathcal{A}$ such that $\{\alpha, \bar{\alpha}\} \subseteq X$, or

- $\mathrm{X}$ is contradictory wrt $\neg,{ }^{19}$ i.e. there exists $\sigma \in \mathcal{L}$ such that $\{\sigma, \neg \sigma\} \subseteq X$.

Definition 16 Let $\mathcal{F}=(\mathcal{R}, \mathcal{A},-)$ be an $A B A$ framework. Define

$$
A L_{\mathcal{F}}=\left(C N_{\mathcal{F}}, C O N T R A_{\mathcal{F}}\right)
$$

where

1. $C N_{\mathcal{F}}=C N_{\mathcal{R}}$.

2. $C O N T R A_{\mathcal{F}}$ is the set of all contradictory sets.

It follows immediately

Lemma $7 A L_{\mathcal{F}}$ is an abstract logic.

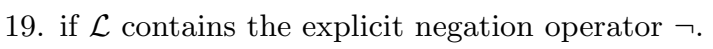


Remark 5 For simplicity, if there are no possibilities for misunderstanding, we often write in this section $C N(S)$ or $C O N T R A$ for $C N_{\mathcal{F}}(S)$ or $C O N T R A_{\mathcal{F}}$ respectively.

We adapt the self-contradiction axiom for assumption-based argumentation below.

Definition 17 Let $\mathcal{F}$ be an $A B A$ framework. We say that the abstract logic $A L_{\mathcal{F}}$ satisfies the

ab-self-contradiction axiom $^{20}$ if for each inconsistent set of assumptions $X$, there is $\alpha \in X$ such that $\bar{\alpha} \in C N_{\mathcal{F}}(X)$.

\subsection{Closure and Consistency in Assumption-Based Argumentation}

We first recall definitions of arguments and attack relation associated to an ABA framework.

Definition 18 1. Any assumption $\alpha$ is an argument whose support and conclusion are $\{\alpha\}, \alpha$ respectively.

2. Let $\sigma_{1}, \ldots \sigma_{n} \rightarrow \sigma$ be a rule. Further suppose that $A_{1}, \ldots, A_{n}$ are arguments with conclusions $\sigma_{1}, \ldots, \sigma_{n}$ respectively. Then $A_{1}, \ldots, A_{n} \rightarrow \sigma$ is an argument whose conclusion is $\sigma$ and whose support is the union of the supports of $A_{1}, \ldots, A_{n}$

3. Every argument is constructed by applying finitely many times the above two steps.

Remark 6 Arguments are often viewed as proof trees. Arguments of the form $A_{1}, \ldots, A_{n} \rightarrow$ $\sigma$ are proof trees with the root labelled by $\sigma$ and the children of the root are the roots of subtrees $A_{1}, \ldots, A_{n}$. Note that if $n=0$, the proof tree consists of just the root. If $A$ is an assumption $\alpha$ then the proof tree consists of just the root labelled by $\alpha$

Notation 3 1. The support of an argument A is denoted by $\operatorname{supp}(A)$.

The support of a set of arguments $S$ is the union of the supports of each individual argument in it and denoted by $\operatorname{supp}(S)$.

2. The conclusion of an argument $\mathrm{A}$ is denoted by $\operatorname{Cnl}(A)$.

Definition 19 1. An argument $A$ attacks an argument $B$ if $C n l(A)=\bar{\alpha}$ for some $\alpha \in$ $\operatorname{supp}(B)$.

2. We say $B$ is a subargument of an argument $A$ of the form $A_{1}, \ldots, A_{k} \rightarrow \alpha$, denoted by $B \sqsubseteq A$, if $B=A$ or $B$ is a subargument of some $A_{i}$.

Remark 7 For simplicity, we identify an assumption-based framework $\mathcal{F}$ with the logicassociated argumentation framework $\left(A F_{\mathcal{F}}, \sqsubseteq, A L_{\mathcal{F}}, C n l\right)$ where $A F_{\mathcal{F}}$ is the argumentation framework generated from $\mathcal{F}$ (according to definitions 18, 19).

It is not difficult to see

Theorem 6 ABA frameworks are compact.

20. ab stands for assumption-based. 
Proof See appendix of section 5 .

Theorem 7 Let $\mathcal{F}$ be an $A B A$ framework. If $A L_{\mathcal{F}}$ satisfies the ab-self-contradiction axiom, then $\mathcal{F}$ is cohesive.

Proof See appendix of section 5.

It follows immediately from theorems 6,7 , corollary 1

Corollary 3 Let $\mathcal{F}$ be an $A B A$ framework. If $A L_{\mathcal{F}}$ satisfies the ab-self-contradiction axiom, then $\mathcal{F}$ satisfies both the closure- and consistency-properties.

\subsection{Logic Programming}

Logic programming could be classified into three different classes of definite programs, normal programs and extended programs with increasing complexity. Bondarenko, Dung, Kowalski and Toni(1997) showed that logic programs are instances of assumption-based argumentation. We discuss below the underlying abstract logics of all classes and the selfcontradiction axiom.

\subsubsection{Definite Logic Programs}

A definite logic program is simply an assumption-based argumentation framework $\mathcal{F}=$ $(\mathcal{R}, \mathcal{A},-)$ based on a language $\mathcal{L}$ where

1. $\mathcal{L}$ consists only of ground atoms and the set of assumptions is empty.

2. Rules in $\mathcal{R}$ are of the form $a_{1}, \ldots, a_{n} \rightarrow h$ where $h, a_{1}, \ldots, a_{n}$ are atoms from $\mathcal{L}$.

As there is no contradiction in $\mathcal{L}, C O N T R A_{\mathcal{F}}=\emptyset$. The ab-self-contradiction axiom hold trivially. Since there is no attack between arguments, the only extension is the set of all arguments. The closure and consistency properties hold obviously.

\subsubsection{Normal Logic Programs}

A normal logic program is an assumption-based argumentation framework $\mathcal{F}=(\mathcal{R}, \mathcal{A},-)$ based on a language $\mathcal{L}$ where

1. $\mathcal{L}$ consists of atoms of the form $a, b, \ldots$ together with the negation-as-failure literals of the form not_a where a is an atom.

2. Assumptions are negation-as-failure literals not_a whose contraries are a.

3. Rules in $\mathcal{R}$ are of the form $l_{1}, \ldots, l_{n} \rightarrow h$ where $h$ is an atom and $l_{1}, \ldots, l_{n}$ are literals from $\mathcal{L}$. 
$C O N T R A_{\mathcal{F}}$ consists of all subsets of $\mathcal{L}$ that contain a pair $a$, not_a for some atom a.

The ab-self-contradiction axiom holds obviously. ${ }^{21}$ The closure and consistency properties hence hold for all extensions of normal programs.

\subsubsection{Extended Logic Programs}

An extended logic program (Gelfond \& Lifschitz, 1990; Lifschitz, 1999) is an assumptionbased argumentation framework $\mathcal{F}=(\mathcal{R}, \mathcal{A},-)$ based on a language $\mathcal{L}$ where

1. $\mathcal{L}$ consists of atoms of the form $a, b, \ldots$ and their explicit negations $\neg a, \neg b, \ldots$ together with the negation-as-failure literals of the form not_l where 1 is a classical literal (i.e. an atom or the explicit negation of an atom).

2. Assumptions are negation-as-failure literals not_l whose contraries are 1 .

3. $\mathcal{R}$ consists of rules of the form $l_{1}, \ldots, l_{n} \rightarrow h$ where $h$ is a classical literal and $l_{1}, \ldots, l_{n}$ are literals from $\mathcal{L}$

$C O N T R A_{\mathcal{F}}$ consists of all subsets of $\mathcal{L}$ that contain a pair $a, \neg a$ for some atom a or a pair $l$, not_l for some classical literal $l$.

From theorems 6,7 and corollary 3 , it follows immediately

Corollary 4 Let $\mathcal{F}$ be a extended logic program. If $C N_{\mathcal{F}}(\emptyset)$ is not contradictory wrt $\neg$ and $A L_{\mathcal{F}}$ satisfies the ab-self-contradiction axiom then $\mathcal{F}$ is compact and cohesive and hence satisfies both the closure and consistency properties.

\section{Translating Rule-Based Argumentation into Assumption-Based Argumentation}

We have showed in the previous two sections that the self-contradiction axioms are sufficient and natural conditions for ensuring closure and consistency properties in both assumptionbased and rule-based argumentation.

In this section, we argue that the self-contradiction axiom in rule-based systems is subsumed by the assumption-based self-contradiction axiom by giving a translation from rule-based systems into equivalent assumption-based ones. More generally, the translation suggests that rule-based argument systems (without preferences) are subsumed by assumption-based argumentation.

Let $A S=(R S, R D)$ be an arbitrary but fixed rule-based argumentation system such that for each $r \in R D$, there is no rule in $R S \cup R D$ whose head coincides with $O j(r)$ or whose body contains an occurrence of $O j(r)$. We translate $A S$ into an assumption-based system in the following definition.

Definition $20 \mathcal{T}(A S)=(\mathcal{R}, \mathcal{A},-)$ is defined as follows:

21. We give a short proof here. Let $\mathrm{X}$ be an inconsistent set of assumptions. Hence there is an assumption $\alpha$ s.t. $\{\alpha, \bar{\alpha}\} \subseteq C N_{\mathcal{F}}(X)$. Since assumptions do not appear in the heads of rules, $\alpha \in X$. 
1. $\mathcal{A}=\{O j(r) \mid r \in R D\} \cup\{$ not_l $\mid$ l is the head of some rule in $R D\}$

where $O j(r)$ is viewed as an assumption indicating that rule $r$ is applicable and not_l is a negation-as-failure assumption stating that there is "no evidence-to-the-contrary" of $l$.

2. $\mathcal{R}=R S \cup\{\operatorname{Tr}(r) \mid r \in R D\}$

where $\operatorname{Tr}(r)$ is of the form

$$
O j(r), \text { not_}_{-} h, \lambda_{1}, \ldots, \lambda_{n} \rightarrow h
$$

if $r$ is of the form $\lambda_{1}, \ldots, \lambda_{n} \Rightarrow h$

3. $\overline{O j(r)}=\neg O j(r)$ and $\overline{\text { not_l }}=l$

Remark 8 Since there is no rule in $R S \cup R D$ whose head is of the form $O j(r)$, no assumption in $\mathcal{A}$ coincides with the head of any rule in $\mathcal{R}$. Therefore $C N_{\mathcal{T}(A S)}(\emptyset)$ is not contradictory wrt - . It is not difficult to see that $C N_{\mathcal{T}(A S)}(\emptyset)=C N_{A S}(\emptyset) . C N_{\mathcal{T}(A S)}(\emptyset)$ is hence not contradictory wrt $\neg$. Therefore $C N_{\mathcal{T}(A S)}(\emptyset)$ is not contradictory.

$\mathcal{T}(A S)$ is hence an assumption-based argumentation system.

As $A S$ and $\mathcal{T}(A S)$ are distinct systems, an attentive reader may ask in what sense they are equivalent?

Before giving a formal elaboration on this question, let us look at an example.

Example 9 Consider a simple rule-based system $A S$ consisting of one strict rule and one defeasible rule :

$$
r_{0}: \rightarrow b \quad r_{1}: \quad b \Rightarrow f
$$

There are two arguments here:

$$
A_{1}: \rightarrow b \text { and } A_{2}: A_{1} \Rightarrow f
$$

The arguments do not attack each other. Hence the only complete extension $E$ of $A S$ contains both arguments $A_{1}, A_{2}$.

The corresponding assumption-based system $\mathcal{T}(A S)$ consists of two rules:

$$
\rightarrow b \quad O j\left(r_{1}\right), \text { not }_{-} f f, b \rightarrow f
$$

There are four arguments in the assumption-based system:

$$
B_{1}: \rightarrow b \quad B_{2}: C_{0}, C_{1}, B_{1} \rightarrow f \quad C_{0}: O j\left(r_{1}\right) \quad C_{1}: n o t_{-} \neg f
$$

There are no attacks between these four arguments. The only complete extension $E^{\prime}$ of $\mathcal{T}(A S)$ consists of all four arguments. In fact the information contained in $E^{\prime}$ is fully captured in the set $S=\left\{B_{1}, B_{2}\right\}$ since $C_{0}, C_{1}$ are subarguments of $B_{2}$, hence any attack against 
them is also an attack against $B_{2}$. We could view the set $S$ as an equivalent representative of $E^{\prime}$. S could be viewed as representing the core of $E^{\prime}$.

The equivalence between $E$ and $E^{\prime}$ is captured by the correspondence between arguments $A_{1}, A_{2}$ and arguments $B_{1}, B_{2}$ respectively.

Note that arguments $C_{0}, C_{1}$ in extension $E^{\prime}$ of $\mathcal{T}(A S)$ explicitly represent the implicit meta-level information contained in extension $E$ of $A S$, namely, defeasible rule $r_{1}$ is applicable and there is no argument with conclusion $\neg f$.

Let $A F_{0}=\left(A R_{0}, a t t_{0}\right), A F_{1}=\left(A R_{1}, a t t_{1}\right)$ be the argumentation frameworks corresponding to $A S, \mathcal{T}(A S)$ respectively.

Definition 21 Let $S$ be a set of arguments in $A F_{1}$. The core of $S$, denoted by $\mathbf{C o r e ( S ) ,}$ is defined by

$$
\operatorname{Core}(S)=S \backslash \mathcal{A}
$$

i.e Core(S) contains arguments in $S$ that are not assumptions.

For illustration, in example 9 , Core $\left(E^{\prime}\right)=\left\{B_{1}, B_{2}\right\}$.

Lemma 8 1. Let $S$ be a set of arguments in $A F_{1}$ and $A$ be an argument in $A F_{1}$. It holds: $A$ is acceptable wrt $S$ iff $A$ is acceptable wrt Core $(S)$.

2. Let $S$ be a set of arguments in $A F_{1} . S$ is admissible iff Core $(S)$ is admissible.

Proof See appendix of section 6 .

The following lemma states that complete sets are identified uniquely by their cores.

Lemma 9 Let $E, E^{\prime}$ be complete extensions of $A F_{1}$. It holds:

$$
E=E^{\prime} \text { iff } \operatorname{Core}(E)=\operatorname{Core}\left(E^{\prime}\right)
$$

Proof See appendix of section 6.

We present a bijection between complete extensions in $A F_{0}$ and complete extensions in $A F_{1}$ by defining a natural one-one mapping from $A R_{0}$ into $A R_{1}$ :

Definition 22 Define

$$
\mathcal{C}: A R_{0} \longrightarrow A R_{1}
$$

such that following properties are satisfied:

1. If $A$ is of the form $A_{1}, \ldots, A_{n} \rightarrow h, n \geq 0$, then $\mathcal{C}(A)$ is of the form

$$
\mathcal{C}\left(A_{1}\right), \ldots, \mathcal{C}\left(A_{n}\right) \rightarrow h^{22}
$$

22. Note that if $\mathrm{A}$ is of the form $\rightarrow h, \mathcal{C}(A)=A$. 
2. If $A$ is a basic defeasible argument of the form $A_{1}, \ldots, A_{n} \Rightarrow h$ then $\mathcal{C}(A)$ is of the form

$$
O j(r), \text { not_}_{-} h, \mathcal{C}\left(A_{1}\right), \ldots, \mathcal{C}\left(A_{n}\right) \rightarrow h^{23}
$$

where $r$ is the last rule of $A$.

For a set of arguments $S \subseteq A R_{0}$, let $\mathcal{C}(S)=\{\mathcal{C}(A) \mid A \in S\}$. It follows

Lemma 10 Let $A, B \in A R_{0}$ and $S \subseteq A R_{0}$. The following observations hold:

1. $\operatorname{Cnl}(A)=\operatorname{Cnl}(\mathcal{C}(A))$.

2. $\mathcal{C}$ is an one-one mapping from $A R_{0}$ onto $A R_{1} \backslash \mathcal{A}$

3. $(A, B) \in \operatorname{att}_{0}$ iff $(\mathcal{C}(A), \mathcal{C}(B)) \in a t t_{1}$.

4. $S$ is admissible in $A F_{0}$ if and only if $\mathcal{C}(S)$ is admissible in $A F_{1}$.

5. A is acceptable wrt $S$ iff $\mathcal{C}(A)$ is acceptable wrt $\mathcal{C}(S)$

Proof See appendix of section 6.

Let $\mathcal{L}$ be the language of $A S$ and

$$
\mathcal{L}_{0}=\mathcal{L} \backslash\{O j(r), \neg O j(r) \mid r \text { is a defeasible rule in } A S\}
$$

The equivalence of $A S$ and $\mathcal{T}(A S)$ is established in the following theorem.

Theorem 8 For each complete extension $E$ of $A F_{0}$ there is a complete extension $E^{\prime}$ of $A F_{1}$ and vice versa such that the following properties hold:

1. $\mathcal{C}(E)=\operatorname{Core}\left(E^{\prime}\right)$

2. For each literal $l \in \mathcal{L}_{0}$,

$$
l \in C n l(E) \text { iff } l \in C n l\left(E^{\prime}\right)
$$

Proof See appendix of section 6.

The following theorem shows that the self-contradiction axiom in rule-based argumentation is subsumed by the ab-self-contradiction axiom in assumption-based argumentation.

Let $A L_{i}=\left(C N_{i}, C O N T R A_{i}\right), \mathrm{i}=0,1$, be the abstract logics associated to $A S, \mathcal{T}(A S)$ respectively.

Theorem 9 If $A L_{0}$ satisfies the self-contradiction axiom then $A L_{1}$ satisfies the ab-selfcontradiction axiom.

Proof See appendix of section 6.

23. Note that if $\mathrm{A}$ is a defeasible rule $\mathrm{r}$ of the form $\Rightarrow h, \mathcal{C}(A)$ is of the form $O j(r)$, not $_{-} \neg h, \rightarrow h$. 


\section{Discussion}

Amgoud and Besnard (2009) have introduced the use of Tarski's abstract logic to study the consistency property of logic-based argumentation. In the following, we discuss how compactness and cohesion could be fulfilled in their systems.

As remarked earlier, a Tarski abstract logic is represented by an abstract logic where $C O N T R A \neq \emptyset$ and for each $X \in C O N T R A, C N(X)=\mathcal{L}$ and there is $x \in \mathcal{L}$ such that $C N(\{x\}) \in C O N T R A$.

A Tarski abstract logic is said to be adjunctive if for all $x, y \in \mathcal{L}$, if $C N(\{x\}) \neq$ $C N(\{x, y\}) \neq C N(\{y\})$ then there exists $z$ such that $C N(\{z\})=C N(\{x, y\})$.

A knowledge base is defined as a set $\Sigma \subseteq \mathcal{L}$ such that for each $x \in \Sigma$, $\mathrm{x}$ is consistent.

An argument over $\Sigma$ is a pair $A=(X, \sigma)$ where $\mathrm{X}$ is finite consistent support of $\mathrm{A}$ denoted by $\operatorname{supp}(A)$, and $\sigma \in C N(X)$ is the conclusion of $\mathrm{A}$ denoted by $\operatorname{Cnl}(A)$. The support of a set of arguments is the union of the supports of the individual arguments.

$A R$ denotes the set of all arguments over $\Sigma$.

$X \subseteq \Sigma$ is called a minimal conflict set if $\mathrm{X}$ is inconsistent and each proper subset of $\mathrm{X}$ is consistent. ${ }^{24}$

For the rest of this discussion, we assume that all minimal conflict sets contain two or more elements.

Let att $\subseteq A R \times A R$ be an attack relation.

1. att is said to be context-sensitive iff for all $a, b \in A R$, if $\operatorname{supp}(a) \cup \operatorname{supp}(b)$ is inconsistent then either $(a, b) \in$ att or $(b, a) \in a t t$.

2. att is said to be conflict-dependent iff for all $a, b \in A R$, if $(a, b) \in$ att then $\operatorname{supp}(a) \cup$ $\operatorname{supp}(b)$ is inconsistent.

3. att is said to be symmetric iff for all $a, b \in A R$, if $(a, b) \in$ att then $(b, a) \in$ att.

An argument $\mathrm{B}$ is said to be a subargument of an argument $\mathrm{A}$, denoted $B \sqsubseteq A$ if $B=A$ or $B=(\{\sigma\}, \sigma)$ for some $\sigma \in \operatorname{supp}(A)$.

Lemma 11 If all minimal conflict sets are binary and the attack relation att is contextsensitive, conflict-dependent and symmetric then $L A F=(A F, \sqsubseteq, A L, C n l)$ is a compact and cohesive logic-associated argumentation framework.

Proof See appendix of section 7 .

It follows immediately from theorem 1 :

Corollary 5 If all minimal conflict sets are binary and the attack relation att is contextsensitive, conflict-dependent and symmetric then LAF satisfies both properties of closure and consistency.

24. It is not difficult to see that each minimal conflict set is finite as from $C N(X)=\mathcal{L}$, and $\mathcal{L}=C N(\{x\})$ for some $x \in \mathcal{L}$, it follows that $x \in C N(X)$. From the finiteness axiom, there is a finite subset $\mathrm{Y}$ of $\mathrm{X}$ such that $x \in C N(Y)$. Hence $C N(Y)=\mathcal{L}$. From the minimality of $\mathrm{X}$, it follows $X=Y$. $\mathrm{X}$ is hence finite. 
Corollary 5 is rather limited due to restrictions imposed on the attack relations. This is because the structure of arguments here is rather poor as abstract logics do not reveal any structure of the consequence relation. Our approach of marrying abstract argumentation with abstract logics resulting in logic-associated abstract argumentation addresses this problem by specifying the subargument structure and its relation to the attack relation.

Caminada and Amgoud (2007) have also studied unrestricted rebuts where two arguments with contrary conclusions are considered to attack each other. Defeasible argumentation with attacks based on unrestricted rebuts violates both the consistency and closure properties except for the grounded semantics. Unrestricted rebuts did not gain much attention in the research in assumption-based argumentation or logic programming. This suggests that there are relevant structural features underlying unrestricted attacks that are still not understood. A sensible idea could be to study this kind of attacks within our proposed framework of logic-associated abstract argumentation as it could shed lights on its instances in both defeasible and assumption-based argumentation.

Non-interference, another key rationality postulate for structured argumentation has been proposed by Caminada, Carnielli, and Dunne (2012) and studied extensively by Caminada et al. (2012), and Wu (2012). Non-interference is conceptually different to the consistency and closure properties as the later properties could be viewed as about the correctness of the argument systems while the former is about their structural modularity. As we focus on the correctness of argument systems, a study on their structural modularity is outside the scope of this paper. Nonetheless, non-interference seems to be related to a property of localizing conflicts in arguments systems where we say that an argument system is localized if there is no argument attacking every argument. It is not difficult to see that aspic systems that are closed under contraposition is not localized if there is a rebutting attack in it. In contrast, the self-contradiction axiom allows us to develop localized aspic systems. It would be interesting to see how the two concepts of localized conflicts and non-interference are interrelated.

Toni (2008) has generalized assumption-based argumentation to represent reasoning with both strict and defeasible rules satisfying both the rational properties of logical closure and consistency. As we have showed in section 6, standard assumption-based argumentation captures rule-based argumentation system by a simple and elegant transformation. Hence it is not necessary to generalize assumption-based argumentation to capture defeasible reasoning with strict and defeasible rules. Nevertheless, the proposal by Toni (2008) is interesting. Nielsen and Parson (2007) have also proposed a generalization of abstract argumentation allowing sets of attacking arguments. Prakken (2010) and Modgil and Prakken (2013) had also studied preferences between arguments. It would be interesting to see whether the properties of compactness and cohesion are satisfied in these frameworks.

We believe that the compactness and cohesion and self-contradiction properties could serve as guideline principles in the design of logic-based argumentation systems to ensure the satisfaction of properties of logical closure and consistency. As pointed out by Caminada and Amgoud (2007), there are several argument systems (Garcia \& Simari, 2004; Governatori, Maher, Antoniou, \& Billington, 2004) not satisfying the consistency property. It would be interesting to see how the results in this paper could be applied on them. 


\section{Appendix A. Section 1}

Let $\mathrm{CN}$ be the consequence operator wrt $A S^{\prime}$. It is clear that $C N(\emptyset)=\left\{p, b, \neg O j\left(d_{2}\right), t h\right\}$. Let $c \in C N(X)$ for $c \in \mathcal{L}$ and $X \subseteq \mathcal{L}$. Let $x \in X$. We show that $\neg x \in C N(Y)$ where $Y=X \backslash\{x\} \cup\{\neg c\}$. If $c \in C N(\emptyset)$, from the "absurdity rules", it follows immediately that $\neg x \in C N(Y)$. Suppose $c \notin C N(\emptyset)$. Hence $c \in C N(X)$ iff $c \in X$ or $\mathrm{X}$ contains a pair of literals $a, \neg a$. If $c \in X$ and $x \neq c$, then $c \in Y$. Hence $\{c, \neg c\} \subseteq Y$. From the "absurdity rules", $\mathcal{L}=C N(Y)$. If $c \in X$ and $x=c$ then $\neg x \in Y$. Hence $\neg x \in C N(Y)$. If X contains a pair of literals $a, \neg a$ and $x \notin\{a, \neg a\}$, then $\mathcal{L}=C N(Y)$. If $x \in\{a, \neg a\}$ then $\neg x \in Y$.

\section{Appendix B. Section 3}

Lemma 1 Let $\mathrm{S}$ be a set of arguments. The following assertions hold:

1. For each argument $\mathrm{A}, \mathrm{A}$ is generated by $\{A\}$.

2. $\operatorname{Sub}(S) \subseteq G N(S)$.

3. $C n l(G N(S)) \subseteq C N(C n l(S u b(S)))$.

4. $C N(C n l(G N(S)))=C N(C n l(S u b(S)))$.

5. For each argument $\mathrm{C}, \mathrm{C}$ attacks $G N(S)$ iff $\mathrm{C}$ attacks $\mathrm{S}$.

Proof The first assertion is obvious from definitions 6 and 7. Since for each $A \in S u b(S)$, $\{A\} \subseteq S u b(S)$, it follows immediately from the first assertion that each argument in $\operatorname{Sub}(S)$ is generated by S. The third assertion follow immediately from definitions 6 and 7 . The fourth assertion follows from the second and third ones.

From $S \subseteq G N(S)$, it is clear that if $\mathrm{C}$ attacks $\mathrm{S}$, C attacks $G N(S)$. Suppose $\mathrm{C}$ attacks $G N(S)$. Let $A \in G N(S)$ s.t. C attacks A. Let BA be a base of A such that $B A \subseteq S u b(S)$. $\mathrm{C}$ hence attacks BA. Therefore $\mathrm{C}$ attacks $S u b(S)$. Thus $\mathrm{C}$ attacks S.

Theorem 1 Let E be a complete extension. Then $G N(E)=E$

Proof Since each attack against $G N(E)$ is an attack against E (lemma 1, last assertion), each attack against $G N(E)$ is counterattacked by $\mathrm{E}$ as $\mathrm{E}$ is a complete extension. Therefore $G N(E) \subseteq E$. From second assertion in lemma 1, it follows $E \subseteq G N(E)$. Hence $G N(E)=$ E.

\section{Appendix C. Section 4}

Remark 9 A strict argument over $X \subseteq \mathcal{L}$ is a strict argument over the set of rules $R S \cup\{\rightarrow$ $\alpha \mid \alpha \in X\}$.

Remark 10 For a strict argument $A$ over $X$, the set of premises of $A$, denoted by $\operatorname{Prem}(A)$, is the set of literals from $X$ labelling the leaves of $A$ (viewed as a proof tree).

Theorem 4 Rule-based argumentation systems are compact. 
Proof Let $A S$ be a rule-based system and let $\mathrm{S}$ be a set of arguments wrt $A S$ and $\sigma \in$ $C N_{A S}(C n l(S u b(S)))$. From lemma 2, we only need to show that $\sigma \in C n l(G N(S))$.

Let $X$ be a minimal subset of $C n l(S u b(S))$ such that $\sigma \in C N_{A S}(X)$. Hence there is a strict argument $A_{0}$ over X with conclusion $\sigma$. Further let $S_{X}$ be a minimal set of arguments from $\operatorname{Sub}(S)$ s.t. $\operatorname{Cnl}\left(S_{X}\right)=X$. Let A be the argument obtained by replacing each leaf in $A_{0}$ (viewed as a proof tree) labelled by a literal $\alpha$ from $\mathrm{X}$ by an argument with conclusion

$\alpha$ from $S_{X}$. It is obvious that the conclusion of $\mathrm{A}$ is $\sigma$. We show that $S_{X}$ is a base of A. Suppose B is an argument attacking A. Since $A_{0}$ is a strict argument over X, B must attack a basic defeasible subargument of some argument in $S_{X}$. Hence B attacks $S_{X}$. Thus $A \in G N(S)$. Hence $\sigma \in C n l(G N(S))$. We have proved that that the rule-based argumentation system $A S$ is compact.

Lemma 4 Let $\mathrm{A}$ be an argument and $\mathrm{BD}$ be the set of basic defeasible subarguments of A. Then $C n l(A) \in C N_{A S}(C n l(B D))$.

Proof By induction on the size of A.

Basic Step A is of the form $\rightarrow / \Rightarrow \sigma$.

Suppose A is of the form $\rightarrow \sigma$ then $\sigma \in C N_{A S}(\emptyset)$. From $B D=\emptyset$, the lemma holds. Suppose $\mathrm{A}$ is of the form $\Rightarrow \sigma$ then $B D=\{A\}$. The lemma holds.

Inductive Step. Suppose $\mathrm{A}$ is of the form $A_{1}, \ldots, A_{n} \rightarrow / \Rightarrow \sigma$

Suppose $\mathrm{A}$ is of the form $A_{1}, \ldots, A_{n} \Rightarrow \sigma$ then $A \in B D$. The lemma holds obviously.

Suppose $\mathrm{A}$ is of the form $A_{1}, \ldots, A_{n} \rightarrow \sigma$. Hence $B D$ is the union of the sets $B D_{1}, \ldots, B D_{n}$ of basic defeasible subarguments of $A_{1}, \ldots, A_{n}$ respectively. From the induction hypothesis, $C n l\left(A_{i}\right) \in C N_{A S}\left(C n l\left(B D_{i}\right)\right), 0 \leq i \leq n$. Hence $C n l(A) \in C N_{A S}(C n l(B D))$.

Theorem 5 Suppose $A L_{A S}$ satisfies the self-contradiction axiom. Then $A S$ is cohesive.

Proof Let $\mathrm{S}$ be an inconsistent set of arguments. Hence $C n l(S u b(S))$ is inconsistent. Define $B D$ to be the set of all basic defeasible arguments in $S u b(S)$. It is clear that $B D \neq \emptyset$. From lemma 4, it follows that $\operatorname{Cnl}\left(S u b(S) \subseteq C N_{A S}(C n l(B D))\right.$. Hence $C N_{A S}(C n l(S u b(S)))=$ $C N_{A S}(C n l(B D))$. $C n l(B D)$ is therefore inconsistent. Since $A L_{A S}$ satisfies the self-contradiction axiom, there is $\sigma \in C n l(B D)$ such that $\neg \sigma \in C N_{A S}(C n l(B D))$. Let $B \in B D$ with $C n l(B)=\sigma$. From $C N_{A S}(C n l(S u b(S)))=C N_{A S}(C n l(B D))$, it follows that $\neg \sigma \in C N_{A S}(C n l(S u b(S)))$. From the compactness of $A S$ and $S u b(S) \subseteq G N(S)$, it follows that there is an argument $A \in G N(S)$ such that $\operatorname{Cnl}(A)=\neg \sigma$. Hence $A$ attacks B. Since $B \in B D \subseteq S u b(S) \subseteq$ $G N(S), G N(S)$ is conflicting.

\section{Lemma 5}

1. If AL is closed under contraposition, then AL satisfies the strong absurdity axiom.

2. If AL satisfies the strong absurdity axiom then AL satisfies the self-contradiction axiom.

\section{Proof}


1. Suppose AL is closed under contraposition. Let $X \in C O N T R A$. Hence there is an atom a s.t. $\{a, \neg a\} \subseteq X$. From $a \in C N(\{a, \alpha\})$ for any literal $\alpha$, and from the closure under contraposition property, it follows that $\neg \alpha \in C N(\{a, \neg a\})$. Hence $\neg \alpha \in$ $C N(X)$ for any literal $\alpha$. We have proved that $\mathcal{L}=C N(X)$ for each $X \in C O N T R A$. From definition 10, it follows that AL satisfies the strong absurdity axiom.

2. Suppose AL satisfies the strong absurdity axiom. Let $X \subseteq \mathcal{L}$ such that $\mathrm{X}$ is minimal inconsistent. Therefore $C N(X) \in C O N T R A$. From the idempotence axiom and the strong absurdity axiom, $C N(X)=\mathcal{L}$. It holds obviously: $\neg X \subseteq C N(X)$.

Lemma 6 Let $A S=(R S, R D)$ such that the set of strict rules $R S$ is closed under transposition. Then $A L_{A S}$ satisfies the self-contradiction axiom.

Proof We first prove the following assertion.

Assertion: Let A be a strict argument over $\mathrm{X}$ with conclusion $\sigma$ and $\emptyset \neq \operatorname{Prem}(A) \subseteq X$. Then for each $\alpha \in \operatorname{Prem}(A)$, there is an argument B with premises in $\operatorname{Prem}(A) \cup\{\neg \sigma\}$ and conclusion $\neg \alpha$.

Proof We prove by induction on the height of A (as a proof tree). ${ }^{25}$

If the height of $\mathrm{A}$ is 0 , the theorem is obvious.

Suppose $\mathrm{A}$ is of the form $A_{1}, \ldots, A_{n} \rightarrow \sigma$ where $\operatorname{Cnl}\left(A_{i}\right)=\alpha_{i}$. Let $\alpha \in \operatorname{Prem}(A)$. Without loss of generality, let $\alpha \in \operatorname{Prem}\left(A_{n}\right)$. From the closure under transposition, the rule $\alpha_{1}, \ldots, \alpha_{n-1}, \neg \sigma \rightarrow \neg \alpha_{n}$ also belongs to $R S$. Let $B$ be the argument $A_{1}, \ldots, A_{n-1}, \neg \sigma \rightarrow$ $\neg \alpha_{n}$.

From the induction hypothesis, there is a proof tree $\operatorname{Tr}$ whose premises are in $\operatorname{Prem}\left(A_{n}\right) \cup$ $\left\{\neg \alpha_{n}\right\}$ and whose conclusion is $\neg \alpha$.

Let $T r^{\prime}$ be the tree obtained from $T r$ by replacing each occurrence of premise $\neg \alpha_{n}$ by the argument $B$. It is clear that $\operatorname{Prem}\left(\operatorname{Tr}^{\prime}\right) \subseteq \operatorname{Prem}(A) \cup\{\neg \sigma\}$ and $\operatorname{Cnl}\left(\operatorname{Tr}^{\prime}\right)=\neg \alpha$.

Let $X \subseteq \mathcal{L}$ s.t. $\mathrm{X}$ is minimal inconsistent. Hence there are two arguments $A_{0}, A_{1}$ with premises in $\mathrm{X}$ and conclusions $\sigma, \neg \sigma$ respectively. From the minimality of $\mathrm{X}$, it holds: $X=\operatorname{Prem}\left(A_{0}\right) \cup \operatorname{Prem}\left(A_{1}\right)$. Let $\alpha \in X$. Without loss of generality, suppose $\alpha \in \operatorname{Prem}\left(A_{0}\right)$. From the above assertion, it follows that there exists an argument $B$ with conclusion $\neg \alpha$ and $\operatorname{Prem}(B) \subseteq \operatorname{Prem}\left(A_{0}\right) \cup\{\neg \sigma\}$. Let A be the argument obtained by replacing each leaf labelled by $\neg \sigma$ in $B$ by tree $A_{1}$. It is clear that $\operatorname{Prem}(A) \subseteq X$ and the conclusion of $\mathrm{A}$ is $\neg \alpha$.

\section{Appendix D. Section 5}

Theorem 6 ABA frameworks are compact.

Proof Let S be a set of arguments. Let $S U=S u b(S)$ and $C S U=C n l(S U)$. We only need to prove that $C N(C S U) \subseteq C n l(G N(S)$ ) (lemma 2). Let $\sigma \in C N(C S U)$. It is easy to see that there is an argument A (viewed as a proof tree) with conclusion $\sigma$ whose leaves

25. The height of a proof tree is the length (the number of links) of the longest path from the root to a leaf node. 
are labelled by sentences in $C S U$. Expand this proof tree at each leaf labelled by $\delta \in C S U$ by a proof tree representing an argument in SU with conclusion $\delta$. The new proof tree corresponds to an argument $\mathrm{B}$ with conclusion $\sigma$. The proof trees in $\mathrm{SU}$ that are used to expand A obviously form a base of B. It is hence clear that B is generated by $\mathrm{S}$.

Theorem 7 Let $\mathcal{F}$ be an ABA framework. If $A L_{\mathcal{F}}$ satisfies the ab-self-contradiction axiom, then $\mathcal{F}$ is cohesive.

Proof Let $\mathrm{S}$ be an inconsistent set of arguments. Hence $\operatorname{supp}(S)$ is inconsistent. Since $C N_{\mathcal{F}}$ satisfies the assumption-based self-contradiction axiom, there is $\alpha \in \operatorname{supp}(S)$ such that $\bar{\alpha} \in C N_{\mathcal{F}}(\operatorname{supp}(S))$. From lemma 2, there is an argument $A \in G N(S)$ such that $\operatorname{Cnl}(A)=\bar{\alpha}$. It is obvious that $\mathrm{A}$ attacks any argument in $\mathrm{S}$ whose premises contain $\alpha$. Since $S \subseteq G N(S), G N(S)$ is hence conflicting.

\section{Appendix E. Section 6}

\section{Lemma 8}

1. Let $S$ be a set of arguments in $A F_{1}$ and $\mathrm{A}$ be an argument in $A F_{1}$. It holds:

$\mathrm{A}$ is acceptable wrt $\mathrm{S}$ iff $\mathrm{A}$ is acceptable wrt $\operatorname{Core}(\mathrm{S})$.

2. Let $S$ be a set of arguments in $A F_{1} . \mathrm{S}$ is admissible iff $\operatorname{Core}(S)$ is admissible.

\section{Proof}

1. Since $\operatorname{Core}(S) \subseteq S$, if A is acceptable wrt $\operatorname{Core}(\mathrm{S})$, A is obviously acceptable wrt $\mathrm{S}$. Suppose now that A is acceptable wrt $\mathrm{S}$. Let B attack A. Hence there is $A^{\prime} \in S$ s.t. $A^{\prime}$ attacks B. Therefore $A^{\prime}$ is not an assumption. Hence $A^{\prime} \in \operatorname{Core}(S)$. B is hence attacked by Core $(\mathrm{S})$, i.e. A is acceptable by $\operatorname{Core}(\mathrm{S})$.

2. Follows immediately from the previous assertion.

Lemma 9. Let $E, E^{\prime}$ be complete extensions of $A F_{1}$. It holds:

$$
E=E^{\prime} \text { iff } \operatorname{Core}(E)=\operatorname{Core}\left(E^{\prime}\right)
$$

Proof We only need to show that $\operatorname{Core}(E)=\operatorname{Core}\left(E^{\prime}\right)$ implies $E=E^{\prime}$. The reverse direction is obvious. Let $\operatorname{Core}(E)=\operatorname{Core}\left(E^{\prime}\right)=S$. Let $A \in E \backslash S$. A is hence an assumption acceptable wrt E. From lemma $8, \mathrm{~A}$ is acceptable wrt $\mathrm{S}$. Thus A is acceptable wrt $E^{\prime}$ (lemma 8). Hence $A \in E^{\prime}$. Similarly, we could show that each assumption in $E^{\prime} \backslash S$ belongs to E. We thus proved $E=E^{\prime}$.

Lemma 10 Let $A, B \in A R_{0}$ and $S \subseteq A R_{0}$. The following observations hold:

1. $\operatorname{Cnl}(A)=\operatorname{Cnl}(\mathcal{C}(A))$.

2. $\mathcal{C}$ is an one-one mapping from $A R_{0}$ onto $A R_{1} \backslash \mathcal{A}$ 
3. $(A, B) \in \operatorname{att}_{0}$ iff $(\mathcal{C}(A), \mathcal{C}(B)) \in \operatorname{att}_{1}$.

4. $S$ is admissible in $A F_{0}$ if and only if $\mathcal{C}(S)$ is admissible in $A F_{1}$.

5. $A$ is acceptable wrt $\mathrm{S}$ iff $\mathcal{C}(A)$ is acceptable wrt $\mathcal{C}(S)$

\section{Proof}

1. The first assertion is obvious.

2. It is obvious that there is no argument $\mathrm{A}$ in $A R_{0}$ such that $\mathcal{C}(A) \in \mathcal{A}$.

Viewing an argument in $A R_{0}$ as a proof tree, the height of a tree is defined to be the length (i.e. number of links) of the longest path from the root to a leaf. Let $A R_{0, k}$ be the set of all trees of height $\leq k$ in $A R_{0}$. We prove by induction that $\mathcal{C}$ is one-one on $A R_{0, k}$.

It is obvious that $\mathcal{C}$ is one-one on $A R_{0,0}$. Suppose $\mathcal{C}$ is one-one on $A R_{0, k}$. Let $A, B$ be two different arguments in $A R_{0, k+1}$. If the last rules of $\mathrm{A}, \mathrm{B}$ are different then it is obvious that $\mathcal{C}(A), \mathcal{C}(B)$ are different. Suppose that the last rules of $\mathrm{A}, \mathrm{B}$ are identical. Then $A, B$ respectively have the forms $A_{1}, \ldots, A_{n} \rightarrow / \Rightarrow h, B_{1}, \ldots, B_{n} \rightarrow / \Rightarrow h$. Without loss of generality, we can assume that $A_{1} \neq B_{1}$. Hence from the induction hypothesis, $\mathcal{C}\left(A_{1}\right) \neq \mathcal{C}\left(B_{1}\right)$. Therefore $\mathcal{C}(A) \neq \mathcal{C}(B)$.

It is also straightforward to prove by induction that for each $B \in A R_{1} \backslash \mathcal{A}$, there is $A \in A R_{0}$ such that $\mathcal{C}(A)=B$.

3. (a) Suppose $(A, B) \in a t t_{0}$. Let $B^{\prime}$ be a basic defeasible subargument of $\mathrm{B}$ such that A attacks $\mathrm{B}$ (on $B^{\prime}$ ). There are two cases:

i. $\operatorname{Cnl}(A)=\neg O j\left(\operatorname{Lr}\left(B^{\prime}\right)\right)$ (undercut attack). From $C n l(A)=C n l(\mathcal{C}(A))$, it follows $\operatorname{Cnl}(\mathcal{C}(A))=\neg O j(\operatorname{Lr}(B))$ and $O j(\operatorname{Lr}(B)) \in \operatorname{supp}(\mathcal{C}(B)) . \mathcal{C}(A)$ hence attacks $\mathcal{C}(B)$ wrt $a t t_{1}$.

ii. $\operatorname{Cnl}(A)=\neg h$ for $h=\operatorname{Cnl}\left(B^{\prime}\right)$. Hence $\operatorname{Cnl}(\mathcal{C}(A))=\neg h$ and not_ $\neg h \in$ $\operatorname{supp}(\mathcal{C}(B))$. $\mathcal{C}(A)$ hence attacks $\mathcal{C}(B)$ wrt att $_{1}$.

(b) Suppose $(\mathcal{C}(A), \mathcal{C}(B)) \in a t t_{1}$. There are two cases:

i. $\operatorname{Cnl}(\mathcal{C}(A))=\neg O j(r)$ for some defeasible rule r such that $O j(r) \in \operatorname{supp}(\mathcal{C}(B))$. From $C n l(A)=\operatorname{Cnl}(\mathcal{C}(A))$, it follows $C n l(A)=\neg O j(r)$ and $\mathrm{r}$ is a defeasible rule in $B$. Hence there is basic defeasible subargument $B^{\prime}$ of $\mathrm{B}$ such that $\operatorname{Lr}\left(B^{\prime}\right)=r$. Hence $A$ attacks $\mathrm{B}$ on $B^{\prime}$ in $A F_{0}$.

ii. $\operatorname{Cnl}(\mathcal{C}(A))=\neg h$ such that not_ $\neg h \in \operatorname{supp}(B)$. Hence there is basic defeasible rule $\mathrm{r}$ in $B$ such that $h d(r)=h$. Therefore there is a subargument $B^{\prime}$ of $\mathrm{B}$ such that $\operatorname{Lr}\left(B^{\prime}\right)=r$. Hence $A$ attacks $\mathrm{B}$ on $B^{\prime}$ (by rebutting) in $A F_{0}$.

4. From assertion 3, it is clear that $S$ is conflict-free iff $\mathcal{C}(S)$ is conflict-free.

Suppose $\mathrm{S}$ defends itself against all attacks. Let A attack $\mathcal{C}(S)$ in $A F_{1}$. Therefore $\mathrm{A}$ is not an assumption. From the second assertion, there is $B=\mathcal{C}^{-1}(A)$. From assertion 3 , it follows B attacks $\mathrm{S}$. Therefore $\mathrm{S}$ attacks B. Hence $\mathcal{C}(S)$ attacks $\mathrm{A}$. 
Suppose $\mathcal{C}(S)$ defends itself against all attacks. Let $\mathrm{A}$ attack $\mathrm{S}$ in $A F_{0}$. Let $B=\mathcal{C}(A)$. From assertion 3, it follows B attacks $\mathcal{C}(S)$. Therefore $\mathcal{C}(S)$ attacks B. Hence $\mathrm{S}$ attacks A.

5. Follows immediately from assertion 3.

Theorem 8 For each complete extensions $E$ of $A F_{0}$ there is a complete extension $E^{\prime}$ of $A F_{1}$ and vice versa such that following properties hold:

1. $\mathcal{C}(E)=\operatorname{Core}\left(E^{\prime}\right)$

2. For each literal $l \in \mathcal{L}_{0}, l \in C n l(E)$ iff $l \in C n l\left(E^{\prime}\right)$

Proof Let $\mathrm{E}$ be a complete extension of $A F_{0}$. From assertion 4 in lemma 10, it follows $S=\mathcal{C}(E)$ is admissible. Let $\mathcal{A}_{S}$ be the set of assumptions acceptable wrt $\mathrm{S}$. We show that $E^{\prime}=S \cup \mathcal{A}_{S}$ is complete. Let B in $A F_{1}$ be acceptable wrt $E^{\prime}$. Suppose B is not an assumption. Let $A=\mathcal{C}^{-1}(B)$. From lemma 10, assertion 5, A is acceptable wrt E. Hence $A \in E$. Therefore $B \in S$. If $\mathrm{B}$ is an assumption, $B \in \mathcal{A}_{S}$. We have proved that $E^{\prime}$ is complete and $\operatorname{Core}\left(E^{\prime}\right)=\mathcal{C}(E)$. The uniqueness of $E^{\prime}$ follows directly from the lemma 9 .

Let $l \in \mathcal{L}_{0}$ such that $l \in \operatorname{Cnl}\left(E^{\prime}\right)$. Since $l \notin \mathcal{A}$, it is clear that $l \in \operatorname{Cnl}\left(\operatorname{Core}\left(E^{\prime}\right)\right)$. Hence $l \in \operatorname{Cnl}(\mathcal{C}(E))$. From the first assertion in lemma 10, it follows $l \in C n l(E)$.

Theorem 9 If $A L_{0}$ satisfies the self-contradiction axiom then $A L_{1}$ satisfies the ab-selfcontradiction axiom.

Proof Suppose $A L_{0}$ satisfies the self-contradiction axiom. Let $X \subseteq \mathcal{A}$ be an inconsistent set of assumptions in $\mathcal{T}(A S)$. We want to show that there exists $\alpha \in X$ such that $\bar{\alpha} \in$ $C N_{1}(X)$. Suppose the contrary. It follows immediately that there is atom a such that $\{a, \neg a\} \subseteq C N_{1}(X)$. There are two cases.

Case 1: $\{a, \neg a\} \cap \mathcal{A} \neq \emptyset$. Since $\neg a \notin \mathcal{A}$, a is an assumption. As classical negation does not apply to negation-as-failure literal, it follows $a=O j(d)$ for some $d \in R D$. From $\overline{O j(d)}=\neg a \in C N_{1}(X)$, this is a contradiction to the hypothesis that $\nexists \alpha \in X$ such that $\bar{\alpha} \in C N_{1}(X)$. This case hence can not occur.

Case 2: $\{a, \neg a\} \cap \mathcal{A}=\emptyset$. Therefore $\{a, \neg a\} \cap X=\emptyset$. Let $S_{1}$ be the set of all arguments in $A R_{1} \backslash \mathcal{A}$ whose support is a subset of X. From $\{a, \neg a\} \subseteq C N_{1}(X) \backslash X$, it follows $S_{1} \neq \emptyset$. It is not difficult to see that $\operatorname{Cnl}\left(S_{1}\right)=C N_{1}(X) \backslash X$. Let $S_{0}$ be a set of arguments from $A R_{0}$ such that $S_{0}=\mathcal{C}^{-1}\left(S_{1}\right)$. From $C n l\left(S_{0}\right)=\operatorname{Cnl}\left(S_{1}\right)$, it follows that $\operatorname{Cnl}\left(S_{0}\right)$ is closed (wrt $C N_{0}$ ). It is also easy to see that $S u b\left(S_{0}\right)=S_{0}$. Let $B S$ be the set of basic defeasible arguments in $S_{0}$. From lemma 4, it is clear that $C N_{0}(C n l(B S))=C n l\left(S_{0}\right)$. Since $\{a, \neg a\} \subseteq C N_{1}(X) \backslash X=C n l\left(S_{0}\right)=C N_{0}(C n l(B S)), C n l(B S)$ is hence also inconsistent wrt $A S$. Because $A L_{0}$ satisfies the self-contradiction axiom, there is a literal $h \in C n l(B S)$ such that $\neg h \in C N_{0}(C n l(B S))$. Let $\alpha=n o t_{-} \neg h$. From $h \in C n l(B S)$, it follows $\alpha \in$ $\operatorname{supp}(\mathcal{C}(B S)) \subseteq \operatorname{supp}\left(S_{1}\right)=X$. From $\neg h \in C N_{0}(C n l(B S))=C n l\left(S_{1}\right)=C N_{1}(X) \backslash X$, it follows $\bar{\alpha} \in C N_{1}(X)$. Contradiction.

We have proved that $A L_{1}$ satisfies the assumption-based self-contradiction axiom. 


\section{Appendix F. Section 7}

Lemma 11 If all minimal conflict sets are binary and the attack relation att is contextsensitive, conflict-dependent and symmetric then $L A F=(A F, \sqsubseteq, A L, C n l)$ is a compact and cohesive logic-associated argumentation framework.

Proof To show that $L A F$ is a logic-associated argumentation framework, we need to show that for $A, B, C \in A R$, if $\mathrm{C}$ attacks $\mathrm{B}$ and $B \sqsubseteq A$ then $\mathrm{C}$ attacks $\mathrm{A}$. If $B=A$, there is nothing to prove. Suppose $B=(\{\sigma\}, \sigma)$ for some $\sigma \in \operatorname{supp}(A)$. From the conflict-dependency, it follows $\operatorname{supp}(C) \cup\{\sigma\}$ is inconsistent. Hence $\operatorname{supp}(C) \cup \operatorname{supp}(A)$ is inconsistent. From the context-sensitivity and symmetry of attack relation, it follows $\mathrm{C}$ attacks $\mathrm{A}$.

Let $\mathrm{S}$ be a set of arguments and $\sigma \in C N(C n l(S u b(S)))$. Hence there exists a finite $X \subseteq \operatorname{supp}(S)$ such that $\sigma \in C N(X)$. Let $A=(X, \sigma)$. Let $\mathcal{X}=\{(\{\alpha\}, \alpha) \mid \alpha \in X\}$. It is clear that $\mathcal{X} \subseteq \operatorname{Sub}(S)$. We show that $\mathcal{X}$ is a base of A. Suppose B attacks A. Hence from the conflict-dependency of att, there exists a minimal conflict set $\{\alpha, \beta\}$ such that $\alpha \in \operatorname{supp}(A)$, $\beta \in \operatorname{supp}(B)$. Hence B attacks the argument $(\{\alpha\}, \alpha) \in \mathcal{X}$. Hence $A \in G N(S)$. We have proved $C N(C n l(S u b(S))) \subseteq C n l(G N(S)$. Hence from lemma 2, $A F$ is compact.

Let $\mathrm{S}$ be an inconsistent set of arguments. Hence $\operatorname{supp}(S)$ is inconsistent. There exists a binary minimal conflict set $\{\alpha, \beta\} \subseteq \operatorname{supp}(S)$. Hence arguments $A=(\{\alpha\}, \alpha), B=(\{\beta\}, \beta)$ attack each other. As $A, B \in G N(S), G N(S)$ is conflicting.

\section{References}

Amgoud, L., \& Besnard, P. (2009). Bridging the gap between abstract argumentation systems and logic. In $S U M$, pp. 12-27.

Bondarenko, A., Dung, P., Kowalski, R., \& Toni, F. (1997). An abstract, argumentationtheoretic approach to default reasoning. Artif. Intell., 93, 63-101.

Caminada, M., \& Amgoud, L. (2007). On the evaluation of argumentation formalisms. Artificial Intelligence, 171, 286-310.

Caminada, M., Carnielli, W., \& Dunne, P. (2012). Semi-stable semantics. Journal of Logic and Computation, 22(5), 1207-1254.

Dung, P. M. (1995). On the acceptability of arguments and its fundamental role in nonmonotonic reasoning, logic programming and n-person gamescceptability of arguments and its fundamental role in nonmono- tonic reasoning, logic programming and n-person games. Artif. Intell., $77(2), 321-358$.

Dung, P. M., \& Thang, P. M. (2011). Closure and consistency rationalities in logic-based argumentation. In Balduccini, M., \& Son, T. C. (Eds.), Logic Programming, Knowledge Representation, and Nonmonotonic Reasoning, Vol. 6565, pp. 33-43. Springer.

Garcia, A., \& Simari, G. (2004). Defeasible logic programming: An argumentative approach. TPLP, 4 (1-2), 95-138.

Gelfond, M., \& Lifschitz, V. (1988). The stable model semantics of logic programming. In ICLP/SLP, pp. 579-597. MIT Press. 
Gelfond, M., \& Lifschitz, V. (1990). Logic programs with classical negation. In ICLP, pp. 579-597. MIT Press.

Governatori, G., Maher, M., Antoniou, G., \& Billington, D. (2004). Argumentation semantics for defeasible logic. J. Log. Comput., 14(5), 675-702.

Lifschitz, V. (1999). Answer set planning (abstract). In LPNMR, pp. 373-374. MIT Press.

Modgil, S., \& Prakken, H. (2013). A general account of argumentation with preferences. Artificial Intelligence, 195, 361-397.

Nielsen, S., \& Parson, S. (2007). A generation of dung's abstract framework for argumentation: Arguing with sets of attacking arguments. In $L N C S$, No. 4766, pp. 54-73. Springer Verlag.

Pollock, J. (1995). Cognitive carpentry: A blueprint for how to build a person.. MIT Press, Cambridge MA.

Pollock, J. (1987). Defeasible reasoning. Cognitive Science, 11(4), 481-518.

Prakken, H. (2010). An abstract framework for argumentation with structured arguments. J. Arguments and Computation, 1(2), 93-124.

Prakken, H. (2012). Some reflection on two current trends in formal argumentation. In Artikis, A., Craven, R., Cicekli, N. K., Sadighi, B., \& Stathis, K. (Eds.), Logic Programs, Norms and Action, Vol. 7360 of Lecture Notes in Computer Science, pp. 249-272. Springer Verlag.

Toni, F. (2008). Assumption-based argumentation for closed and consistent defeasible reasoning. JSAI, 390-402.

Wu, Y. (2012). Between Arguments and Conclusions. Ph.D. thesis, University of Luxemburg. 